PROCEEDINGS OF THE

AMERICAN MATHEMATICAL SOCIETY

Volume 132, Number 4, Pages 1049-1058

S 0002-9939(03)07281-2

Article electronically published on November 25, 2003

\title{
ON SOME SPECIAL POLYNOMIALS
}

\author{
V. V. KARACHIK \\ (Communicated by Carmen C. Chicone)
}

\begin{abstract}
New special functions called $G$-functions are introduced. Connections of $G$-functions with the known Legendre, Chebyshev and Gegenbauer polynomials are given. For $G$-functions the Rodrigues formula is obtained.
\end{abstract}

\section{INTRODUCTION}

Let $L_{1}, L_{2}$ be linear partial differential operators acting on functions that belong to the vector space $\mathcal{X}$ such that $L_{k} \mathcal{X} \subset \mathcal{X}(k=1,2)$ and defined in some domain $\Omega \subset \mathbb{R}^{n}$. Denote $\widetilde{\mathbb{N}}=\mathbb{N} \cup\{0\}$.

Definition ([2]). An infinite ordered system of functions $\left\{f_{k}(x) \mid k \in \widetilde{\mathbb{N}}\right\}\left(f_{k} \in \mathcal{X}\right)$ is called $f$-normalized with respect to $\left(L_{1}, L_{2}\right)$ in the domain $\Omega$, having a base $f_{0}(x)$ if everywhere in that domain, $L_{1} f_{0}(x)=f(x)$ and $L_{1} f_{k}(x)=L_{2} f_{k-1}(x)$ for $k \in \mathbb{N}$.

The main property of a system of functions $f$-normalized with respect to operators $\left(L_{1}, L_{2}\right)$ in the domain $\Omega$ is the following: the series $u(x)=\sum_{k=0}^{\infty} f_{k}(x)$ satisfies formally the equation $L_{1} u(x)-L_{2} u(x)=f(x)$ in $\Omega$ and therefore $u(x)$ can be considered as a formal solution to that equation.

Example 1. Let $n=2, L_{1}=y \partial / \partial x, L_{2}=\partial / \partial y$ and $\Omega=\mathbb{R}^{2} /\{y=0\}$. Then a system of functions 0 -normalized with respect to these operators in $\Omega$ can be given in the form $f_{k}(x, y)=\frac{m}{1} \frac{m-2}{2} \cdots \frac{m-2 k+2}{k} y^{m-2 k} x^{k}$ for $k=0,1, \ldots$ and $m \in \mathbb{N}$.

Using the main property of $f$-normalized systems we can easily construct formal solutions to the equation $y u_{x}(x, y)-u_{y}(x, y)=0$ in $\Omega$. In this case formal solutions

$$
u_{m}(x, y)=\sum_{k=0}^{\infty} \frac{(m / 2-k+1)_{k}}{(1)_{k}}(2 x)^{k} y^{m-2 k}, \quad m \in \mathbb{N}
$$

for some $x, y\left(2 x<y^{2}\right)$ are real solutions because the corresponding series are convergent at these points. For even $m$ we get polynomial solutions.

A very important particular case of the above notion is the case when $L_{2}=I$, where $I$ is the identity operator. Then the system of functions $\left\{f_{k}(x) \mid k \in \widetilde{\mathbb{N}}\right\}$

Received by the editors November 5, 2002.

2000 Mathematics Subject Classification. Primary 33D45; Secondary 31B05, 35C05, 33D50.

Key words and phrases. Harmonic polynomials, orthogonal polynomials. 
$f$-normalized with respect to $\left(L_{1}, I\right)$ is called $f$-normalized with respect to $L_{1}$. Elements of the system $\left\{f_{k}(x) \mid k \in \widetilde{\mathbb{N}}\right\}$ in this case satisfy in $\Omega$ the conditions

$$
L_{1} f_{0}(x)=f(x) ; \quad L_{1} f_{k}(x)=f_{k-1}(x), \quad k \in \mathbb{N} .
$$

Example 2. Let $L_{1}(D)=\Delta$. The system $\left\{h_{k}^{s}(x) \mid k \in \widetilde{\mathbb{N}}\right\}$, where

$$
h_{k}^{s}(x)=\frac{|x|^{2 k}}{(2,2)_{k}(n+2 s, 2)_{k}} H_{s}(x), \quad|x|^{2}=x_{1}^{2}+\cdots+x_{n}^{2},
$$

$(a, b)_{k}=a(a+b) \ldots(a+k b-b)$, with the convention $(a, b)_{0}=1$ (here $(a, 1)_{k}=$ $(a)_{k}$; see [5]), and $H_{s}(x)$ is a homogeneous harmonic polynomial of order $s$, is 0normalized with respect to $\Delta$ in $\mathbb{R}^{n}$. It follows from the equality $\Delta\left(H_{s}(x)|x|^{m}\right)=$ $m\left((m+n-2) H_{s}(x)+2 \sum_{i=1}^{n} x_{i} D_{x_{i}} H_{s}(x)\right)|x|^{m-2}$.

Indeed, using homogeneity of $H_{s}(x)$ we can rewrite the above equality in the form $\Delta\left(H_{s}(x)|x|^{m}\right)=m(m+2 s+n-2) H_{s}(x)|x|^{m-2}$. If we take here $m=2 k$, we get

$$
\Delta\left(\frac{|x|^{2 k}}{2 k(n+2 s+2 k-2)} H_{s}(x)\right)=H_{s}(x)|x|^{2(k-1)} .
$$

Dividing both sides by $(2,2)_{k-1}(n+2 s, 2)_{k-1}$, we get (1) for $L_{1}=\Delta, f(x)=0$ and $f_{k}(x)=h_{k}^{s}(x)$, i.e., $\Delta h_{k}^{s}(x)=h_{k-1}^{s}(x)$ for $k>0$ and $\Delta h_{0}^{s}(x) \equiv \Delta H_{s}(x)=0$. A very important detail here is that the base of the system $\left\{h_{k}^{s}(x) \mid k \in \widetilde{\mathbb{N}}\right\}$ is an arbitrary homogeneous harmonic polynomial $H_{s}(x)$.

In a straightforward way from the definition we can establish that the system $\left\{L_{2}^{k} f_{k}(x) \mid k \in \widetilde{\mathbb{N}}\right\}$ is $f$-normalized with respect to $\left(L_{1}, L_{2}\right)$ in domain $\Omega$ if $\forall k, L_{1} L_{2} f_{k}(x)=L_{2} L_{1} f_{k}(x)$ in $\Omega$. Therefore the series

$$
u(x)=\sum_{k=0}^{\infty} L_{2}^{k} f_{k}(x)
$$

is a formal solution to the equation $L(D) u \equiv L_{1} u(x)-L_{2} u(x)=f(x)$ in $\Omega$.

Example 3. Let $L(D)=\Delta$. Using the above construction we are going to find a relation between harmonic polynomials of $n$ variables and harmonic polynomials of $n-1$ variables. There are two possibilities that use (2).

I. Let us choose $L_{1}(D)=\Delta-\partial^{2} / \partial x_{n}^{2} \equiv \widetilde{\Delta}$ and $L_{2}(D)=-\partial^{2} / \partial x_{n}^{2}$. To use (2) we have to find a system of polynomials 0 -normalized with respect to $\widetilde{\Delta}$. We can take it from Example 2. The system $\left\{h_{k}^{s}\left(x_{(n-1)}\right) x_{n}^{m, !} \mid k \in \widetilde{\mathbb{N}}\right\}$, where $x_{(n-1)}=$ $\left(x_{1}, \ldots, x_{n-1}\right), t^{m, !}=t^{m} / m$ ! could be such a system. Therefore, using (2) we can get a harmonic polynomial of $n$ variables in the form

$$
u(x)=\sum_{k=0}^{[m / 2]}(-1)^{k} \frac{\left|x_{(n-1)}\right|^{2 k} x_{n}^{m-2 k, !}}{(2,2)_{k}(n-1+2 s, 2)_{k}} H_{s}\left(x_{(n-1)}\right) .
$$

If we denote

$$
G_{k}^{s}\left(x_{(n)}\right)=\sum_{i=0}^{[k / 2]}(-1)^{i} \frac{\left|x_{(n-1)}\right|^{2 i} x_{n}^{k-2 i, !}}{(2,2)_{i}(n-1+2 s, 2)_{i}},
$$


then we can assert that multiplication of a homogeneous harmonic polynomial $H_{s}\left(x_{(n-1)}\right)$ of $n-1$ variables on the polynomial $G_{k}^{s}\left(x_{(n)}\right)$ gives a harmonic polynomial of $n$ variables, $u(x)=G_{k}^{s}\left(x_{(n)}\right) H_{s}\left(x_{(n-1)}\right)$. It is proved [2] that any homogeneous harmonic polynomial of $n$ variables can be represented in this way.

II. Let us now choose $L_{1}(D)=\partial^{2} / \partial x_{n}^{2}$ and $L_{2}(D)=-\widetilde{\Delta}$. Then the system $\left\{(-1)^{k}\left(H_{0}(\tilde{x}) x_{n}^{2 k, !}+H_{1}(\tilde{x}) x_{n}^{2 k+1, !}\right)\right\}$, where $H_{0}(\tilde{x})$ and $H_{1}(\tilde{x})$ are arbitrary polynomials in $\tilde{x}=\left(x_{1}, \ldots, x_{n-1}\right)$ is 0 -normalized with respect to the operator $L_{2}$ in $\mathbb{R}^{n}$. Using (2) we obtain harmonic polynomials in the known form 6 ]

$$
u(x)=\sum_{k=0}^{\infty}(-1)^{k} x_{n}^{2 k, !} \widetilde{\Delta}^{k} H_{0}(\tilde{x})+\sum_{k=0}^{\infty}(-1)^{k} x_{n}^{2 k+1, ! \widetilde{\Delta}^{k}} H_{1}(\tilde{x}) .
$$

\section{G-POLYNOMiALS}

According to Example 3 the following definition is useful.

Definition. A polynomial $G_{k}^{s}\left(x_{(n)}\right)$ of the form (3) is called a $G$-polynomial of degree $k$, order $s$, and kind $n$.

If $n=2$ there are only two linearly independent homogeneous harmonic polynomials of degree $k(k>0)$ and according to our construction (Example 3, case I) we can write them in the following form:

$$
H_{k}^{s}\left(x_{(2)}\right)=G_{k-s}^{s}\left(x_{(2)}\right) x_{1}^{s}=\sum_{j=0}^{[(k-s) / 2]}(-1)^{j} x_{1}^{2 j+s, !} x_{2}^{k-2 j-s, !}, \quad s=0,1
$$

for $k \in \mathbb{N}$, and with the convention $H_{0}^{0} \equiv 1$. It is necessary to note that $H_{k}^{0}\left(x_{(2)}\right)=$ $\operatorname{Re}\left(x_{2}+i x_{1}\right)^{k, !}$ and $H_{k}^{1}\left(x_{(2)}\right)=\operatorname{Im}\left(x_{2}+i x_{1}\right)^{k, !}$.

This approach allows us to construct harmonic polynomials as a product of $G$ polynomials in the form

$$
G_{(\nu)}\left(x_{(n)}\right)=G_{\nu_{1}-\nu_{2}}^{\nu_{2}}\left(x_{(n)}\right) \cdots G_{\nu_{n-1}-\nu_{n}}^{\nu_{n}}\left(x_{(2)}\right) x_{1}^{\nu_{n}},
$$

where $\nu \in \widetilde{\mathbb{N}}^{n}, \nu_{1} \geq \cdots \geq \nu_{n}$, and $\nu_{n}=0,1$.

In [3] it is proved that polynomials $G_{(\nu)}$ make up a basis among homogeneous harmonic polynomials of degree $\nu_{1}$ and they are orthogonal in $L_{2}\left(\partial S_{n}\right)$, where $S_{n}$ is the unit ball in $\mathbb{R}^{n}$. Moreover, if we denote by $\mathcal{P}$ the set of all polynomials over $\mathbb{C}$, with the scalar product $\langle P(x), Q(x)\rangle=P(D) Q(x)_{\mid x=0}$ (see [1), then polynomials (5) are orthogonal in $\mathcal{P}$ too.

We need also two results from 3 .

Lemma 1. G-polynomials of the same order $s$ and kind $n$ are orthogonal with weight $\rho_{n}^{s}(x)=\left(1-x_{n}^{2}\right)^{s}$ on $\partial S_{n}$.

Lemma 2. Let $f \in C\left(\partial S_{n}\right)$ be taken in the form $f(x)=\varphi\left(|\tilde{x}|, x_{n}\right) P_{k}(\tilde{x})$, where $P_{k}(\tilde{x})$ is a homogeneous polynomial of degree $k$ and $\varphi \in C\left(\partial S_{n}\right)$. Then

$$
\int_{|x|=1} f(x) d x=\frac{1}{\omega_{n-1}} \int_{|x|=1} \varphi\left(|\tilde{x}|, x_{n}\right)\left(1-x_{n}^{2}\right)^{k / 2} d x \int_{|\tilde{x}|=1} P_{k}(\tilde{x}) d \tilde{x} .
$$




\section{Connection of $G$-Functions With LEGENDRE AND CheByshev POLYNOMials}

Let us consider the trace of a $G$-polynomial on the unit sphere. In this case a new notion of $G$-function can be presented.

Definition. The function

$$
G_{k}^{s, n}(t)=\sum_{i=0}^{[(k-s) / 2]}(-1)^{i} \frac{t^{k-s-2 i, !}\left(1-t^{2}\right)^{i+s / 2}}{(2,2)_{i}(n-1+2 s, 2)_{i}}
$$

is called the $G$-function of degree $k$, order $s$, and kind $n$.

It is not difficult to derive from (5) that

$$
G_{(\nu)}(x)=|x|^{\nu_{1}} \prod_{i=1}^{n-1} G_{\nu_{i}}^{\nu_{i+1}, n-i+1}\left(\cos \varphi_{n-i+1}\right),
$$

where $\varphi_{i}=\arccos \left(x_{i} /\left|x_{(i)}\right|\right), \nu_{1} \geq \cdots \geq \nu_{n}$, and $\nu_{n}=0,1$.

Theorem 1. For a G-function of odd kind, the equality

$$
G_{k}^{s, 2 m+3}(t)=\frac{2(s+m) ! !}{(k+s+2 m) !}\left(1-t^{2}\right)^{-m / 2} P_{k+m}^{s+m}(t)
$$

holds, where $m \geq 0, k \geq s \geq 0$, and $P_{k}^{s}(t)$ is the associated Legendre function [4].

Proof. First let us prove that

$$
D_{t}^{m}=\left.m ! \sum_{k=0}^{[m / 2]}(\sqrt{y})^{m-2 k, !} \frac{\left(2 D_{y}\right)^{m-k}}{(2,2)_{k}}\right|_{y=t^{2}} .
$$

For this purpose assume that for some $m \geq 1$ the equality

$$
\left(\sqrt{y} D_{y}\right)^{m}=\sum_{k=0}^{[m / 2]} a_{k}^{m}(\sqrt{y})^{m-2 k, !} D_{y}^{m-k}
$$

holds. Of course for $m=1$ the above equality is true and $a_{0}^{1}=1$.

Applying the operator $\sqrt{y} D_{y}$ to the right-hand side of (9) for even $m$ and assuming that $a_{-1}^{m}=0$, we can obtain

$$
\sum_{k=0}^{[m / 2]}\left(\frac{1}{2} a_{k-1}^{m}+(m-2 k+1) a_{k}^{m}\right)(\sqrt{y})^{m-2 k+1, !} D_{y}^{m-k+1} .
$$

Let us consider the case when $m$ is odd. Then the right-hand side of (9) after applying the operator $\sqrt{y} D_{y}$ takes the form

$$
\sum_{k=0}^{[m / 2]+1}\left(\frac{1}{2} a_{k-1}^{m}+(m-2 k+1) a_{k}^{m}\right)(\sqrt{y})^{m-2 k+1, !} D_{y}^{m-k+1} .
$$

Because the function

$$
\gamma(m)= \begin{cases}{[m / 2],} & m \text { even } \\ {[m / 2]+1,} & m \text { odd }\end{cases}
$$




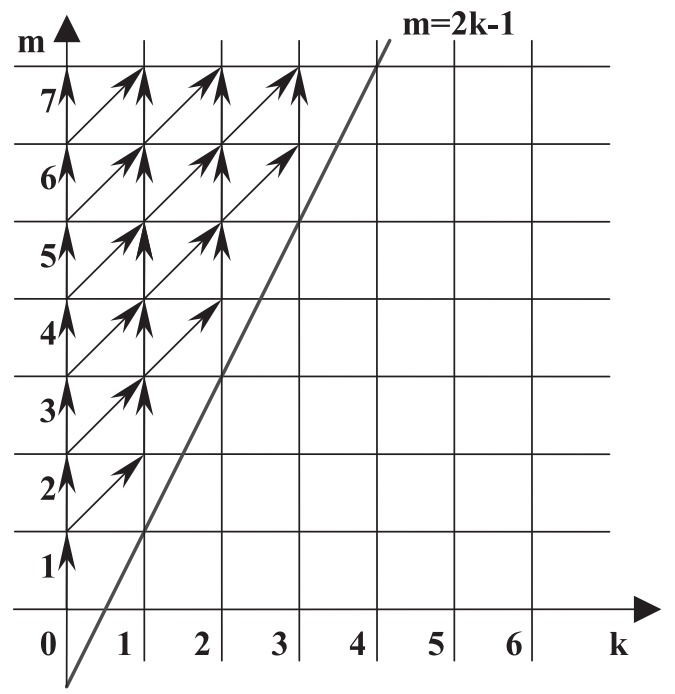

FIGURE 1.

can be written in the form $\gamma(m)=[(m+1) / 2]$, the results are the same for even and odd $m$. Therefore for any $m \geq 1$,

$$
\left(\sqrt{y} D_{y}\right)^{m+1}=\sum_{k=0}^{[(m+1) / 2]}\left(\frac{1}{2} a_{k-1}^{m}+(m-2 k+1) a_{k}^{m}\right)(\sqrt{y})^{m-2 k+1, !} D_{y}^{m-k+1} .
$$

Therefore, for (9) to hold for $m=m+1$, the coefficients $a_{k}^{m}$ should satisfy the following recurrence relation: $a_{k}^{m+1}=\frac{1}{2} a_{k-1}^{m}+(m-2 k+1) a_{k}^{m}$ for $k=0,1$, $\ldots,[(m+1) / 2]$, where $a_{-1}^{m}=0$ and $a_{0}^{1}=1$.

In Figure 1 the obtained recurrence relation is illustrated. The incoming arrows to the point $(k, m)$ show the dependencies of the value $a_{k}^{m}$ on the values of $a_{k-1}^{m-1}$ and $a_{k}^{m-1}$ if they are nonzero. We do not need to consider $a_{k}^{m}$ on the line $m=2 k-1$ because the coefficient for it on this line is $m-2 k+1$, i.e., zero. It is clear that because of $a_{-1}^{m}=0$ we have $a_{0}^{m+1}=(m+1) a_{0}^{m}=(m+1) ! a_{0}^{1}=(m+1)$ !. Therefore $a_{k}^{m}$ is found on the $m$-axis. Now we can find $a_{k}^{m}$ for $k=1$ and $m \geq 2$. In general, the coefficients $a_{k}^{m}$ on the line $k=$ const are fully determined by the $a_{k}^{m}$ on the line $k=$ const -1 . Therefore the solution of the above recurrence relation exists and is unique.

It is not so important here to show how to find this solution. We just take it in the form $a_{k}^{m}=m ! /\left(4^{k} k !\right)$. Let us check it:

$$
\begin{aligned}
\frac{1}{2} a_{k-1}^{m}+(m-2 k+1) a_{k}^{m} & =\frac{m !}{4^{k-1}(k-1) !}\left[\frac{1}{2}+\frac{(m-2 k+1)}{4 k}\right] \\
& =\frac{(m+1) !}{4^{k} k !}=a_{k}^{m+1} .
\end{aligned}
$$

Substituting the obtained value of $a_{k}^{m}$ into (9) and taking into account that $D_{t}=$ $2 \sqrt{y} D_{y}$ we get $(8)$. 
Now we can apply the operator equality (8) for $m=m+s$ to the function $\left(1-t^{2}\right)^{m}$. This yields

$$
\begin{aligned}
D_{t}^{m+s}\left(1-t^{2}\right)^{m} & =\frac{(-1)^{m}(m+s) !(2,2)_{m}}{(2,2)_{s}} \\
& \cdot \sum_{k=0}^{[(m-s) / 2]}(-1)^{k} t^{m-s-2 k, !} \frac{\left(1-t^{2}\right)^{k}}{(2,2)_{k}(2+2 s, 2)_{k}} .
\end{aligned}
$$

From here, using equalities $P_{k}^{s}(t)=\left(1-t^{2}\right)^{s / 2} D_{t}^{s} P_{k}(t), P_{k}(t)=2^{-k} D_{t}^{k}\left(t^{2}-1\right)^{k, !}$, which give us $P_{k}^{s}(t)=\frac{(-1)^{k}}{2^{k} k !}\left(1-t^{2}\right)^{s / 2} D_{t}^{k+s}\left(1-t^{2}\right)^{k}$, we obtain

$$
G_{k}^{s, 3}(t) \equiv \sum_{i=0}^{[(k-s) / 2]}(-1)^{i} t^{k-s-2 i, !} \frac{\left(1-t^{2}\right)^{i+s / 2}}{(2,2)_{i}(2+2 s, 2)_{i}}=\frac{2 s ! !}{(k+s) !} P_{k}^{s}(t) .
$$

Taking into account that $G_{k}^{s, 2 m+3}(t)=\left(1-t^{2}\right)^{-m / 2} G_{k+m}^{s+m, 3}(t)$ we complete the proof.

Consider by analogy with the associated Legendre functions, the associated Chebyshev functions $T_{k}^{s}(t) \equiv\left(1-t^{2}\right)^{s / 2} D_{t}^{s} T_{k}(t)$, where $0<s \leq k, T_{k}^{0}(t)=T_{k}(t)$ being the Chebyshev polynomials.

Theorem 2. For G-functions of even kind the following equality holds:

$$
G_{k}^{s, 2 m+2}(t)=\frac{(2 s+2 m-1) ! !}{(k+s+2 m-1) !(k+m)}\left(1-t^{2}\right)^{-m / 2} T_{k+m}^{s+m}(t),
$$

where $m \geq 0, k \geq s>0$, and $G_{k}^{0,2}(t)=1 / k ! T_{k}^{0}(t)$.

Proof. First we prove that for $s>0$ the following equality holds:

$$
\begin{aligned}
& \left(\sqrt{y} D_{y}\right)^{s} \sum_{i=0}^{[k / 2]}(-1)^{i}(\sqrt{y})^{k-2 i, !} \frac{(1-y)^{i}}{(2,2)_{i}(1,2)_{i}} \\
& \quad=\frac{2^{-s}(k+s-1) !}{(k-1) !(2 s-1) ! !} \sum_{i=0}^{[(k-s) / 2]}(-1)^{i}(\sqrt{y})^{k-s-2 i, !} \frac{(1-y)^{i}}{(2,2)_{i}(1+2 s, 2)_{i}} .
\end{aligned}
$$

Using the property of $\gamma(k)$ functions again (see proof of (9)) for $s \geq 0$, we can get

$$
\begin{aligned}
& \left(\sqrt{y} D_{y}\right) \sum_{i=0}^{[(k-s) / 2]}(-1)^{i}(\sqrt{y})^{k-s-2 i, !} \frac{(1-y)^{i}}{(2,2)_{i}(1+2 s, 2)_{i}} \\
& =\frac{1}{2} \sum_{i=0}^{[(k-s-1) / 2]}(-1)^{i}\left(1+\frac{k-s-2 i-1}{1+2 s+2 i}\right)(\sqrt{y})^{k-s-1-2 i, !} \frac{(1-y)^{i}}{(2,2)_{i}(1+2 s, 2)_{i}} \\
& \quad=\frac{k+s}{2(1+2 s)} \sum_{i=0}^{[(k-s-1) / 2]}(-1)^{i}(\sqrt{y})^{k-s-1-2 i, !} \frac{(1-y)^{i}}{(2,2)_{i}(1+2(s+1), 2)_{i}} .
\end{aligned}
$$

This implies that (11) is true for all $s \geq 1$. Taking $y=t^{2}$, which means $D_{t}=$ $2 \sqrt{y} D_{y}$, we can write (11) in the form

$$
D_{t}^{s} G_{k}^{0,2}(t)=\frac{(k+s-1) !}{(k-1) !(2 s-1) ! !}\left(1-t^{2}\right)^{-s / 2} G_{k}^{s, 2}(t) .
$$


It is not difficult to see that $G_{k}^{0,2}(t)=\frac{1}{k !} \cos (k \arccos t)=\frac{1}{k !} T_{k}(t)$. Therefore $G_{k}^{s, 2}(t)$ can be written in the form

$$
G_{k}^{s, 2}(t)=\frac{(k-1) !(2 s-1) ! !}{(k+s-1) !} \frac{1}{k !}\left(1-t^{2}\right)^{s / 2} D_{t}^{s} T_{k}(t)=\frac{(2 s-1) ! !}{k(k+s-1) !} T_{k}^{s}(t) .
$$

To complete the proof we note $G_{k}^{s, 2 m+2}(t)=\left(1-t^{2}\right)^{-m / 2} G_{k+m}^{s+m, 2}(t)$.

Remark. Using (8) we can obtain $T_{k}(t)=\frac{(-1)^{k}}{(2 k-1) ! !}\left(1-t^{2}\right)^{1 / 2} D_{t}^{k}\left(1-t^{2}\right)^{k-1 / 2}$.

Theorem 3. For G-functions the Rodrigues formula (see [4])

$$
(-1)^{k} D_{t}^{k}\left(1-t^{2}\right)^{k+s+(n-3) / 2}=k !(n-1+2 s, 2)_{k}\left(1-t^{2}\right)^{(s+n-3) / 2} G_{k+s}^{s, n}(t)
$$

holds, and the following connection of G-functions with Gegenbauer polynomials is valid:

$$
G_{k}^{0, n}(t)=\frac{(n-3) !}{(n-3+k) !} C_{k}^{n / 2-1}[t], \quad n \geq 3
$$

Proof. By analogy with (10) and with the help of (8) we can write

$$
\begin{aligned}
D_{t}^{k}\left(1-t^{2}\right)^{k+s+(n-3) / 2} & =k ! \sum_{i=0}^{[k / 2]} t^{k-2 i, !} \frac{2^{k} D_{t^{2}}^{k-i}}{(2,2)_{i}}\left(1-t^{2}\right)^{k+s+(n-3) / 2} \\
& =(-1)^{k} k !(n-1+2 s, 2)_{k}\left(1-t^{2}\right)^{(s+n-3) / 2} G_{k+s}^{s, n}(t),
\end{aligned}
$$

which proves (13). To prove (14) we use the following representation of Gegenbauer polynomials [5]

$$
C_{n}^{\nu}(t)=\sum_{i=0}^{[n / 2]} \frac{(2 \nu)_{n} t^{n-2 i}\left(t^{2}-1\right)^{i}}{2^{2 i} i !(\nu+1 / 2)_{i}(n-2 i) !}
$$

which leads to

$$
C_{n}^{\nu}(t)=(2 \nu)_{n} \sum_{i=0}^{[n / 2]}(-1)^{i} t^{n-2 i, !} \frac{\left(1-t^{2}\right)^{i}}{(2,2)_{i}(1+2 \nu, 2)_{i}}=(2 \nu)_{n} G_{n}^{0,2 \nu+2}(t),
$$

and then $G_{k}^{0, n}(t)=\frac{1}{(n-2)_{k}} C_{k}^{n / 2-1}(t)=\frac{(n-3) !}{(n-3+k) !} C_{k}^{n / 2-1}(t)$ for $n>2$. Proof is completed.

\section{Orthogonality of $G$-FunCtions}

Theorem 4. G-functions of kind $n(n \geq 2)$ and the same order are orthogonal on $[-1,1]$ with weight $\rho(t)=\left(1-t^{2}\right)^{(n-3) / 2}$, and

$$
\int_{-1}^{1}\left(G_{k}^{s, n}(t)\right)^{2}\left(1-t^{2}\right)^{(n-3) / 2} d t=\frac{2^{2 s+n-3} \Gamma^{2}(s+(n-1) / 2)}{(k+(n-2) / 2)(k+s+n-3) !(k-s) !} .
$$

Proof. Using Lemma 1 and because of equality $G_{k}^{s}(x)=G_{k}^{s}\left(|\tilde{x}|, x_{n}\right)$ we obtain

$$
0=\int_{|x|=1} G_{k}^{s}(x) G_{m}^{s}(x)\left(1-x_{n}^{2}\right)^{s} d x=\omega_{n-1} \int_{-1}^{1} G_{k+s}^{s, n}(t) G_{m+s}^{s, n}(t) \rho(t) d t .
$$


This implies orthogonality of $G$-functions $G_{k+s}^{s, n}(t)$ and $G_{m+s}^{s, n}(t)$ with weight $\rho(t)$. From Theorem 1 for odd $n \geq 3$ we get

$$
G_{k}^{s, n}(t)=\frac{(2 s+n-3) ! !}{(k+s+n-3) !}\left(1-t^{2}\right)^{-(n-3) / 4} P_{k+(n-3) / 2}^{s+(n-3) / 2}(t),
$$

and from Theorem 2 for even $n \geq 3$, we have

$$
G_{k}^{s, n}(t)=\frac{(2 s+n-3) ! !}{(k+(n-2) / 2)(k+s+n-3) !}\left(1-t^{2}\right)^{-(n-2) / 4} T_{k+(n-2) / 2}^{s+(n-2) / 2}(t) .
$$

It is known that $\int_{-1}^{1}\left(P_{k}^{s}(t)\right)^{2} d t=\frac{2}{2 k+1} \frac{(k+s) !}{(k-s) !}$ and therefore for odd $n \geq 3$, we have

$$
\int_{-1}^{1}\left(G_{k}^{s, n}(t)\right)^{2} \rho(t) d t=\frac{((2 s+n-3) ! !)^{2}}{(k+(n-2) / 2)(k+s+n-3) !(k-s) !} .
$$

Let $n \geq 2$ be even. Since in this case $G_{k}^{s, n}(t)$ are connected with Chebyshev polynomials, we need to investigate $T_{k}^{s}(t)$. According to the definitions of $T_{k}^{s}(t)$ and $G_{k}^{s, n}(t)$ and with the help of (12) we get

$$
T_{k}^{s}(t)=\frac{k(k+s-1) !}{(2 s-1) ! !}\left(1-t^{2}\right)^{s / 2} \sum_{i=0}^{[(k-s) / 2]}(-1)^{i} \frac{t^{k-s-2 i, !}\left(1-t^{2}\right)^{i}}{(2,2)_{i}(n-1+2 s, 2)_{i}} .
$$

If we use representation (15) (see [5]) we obtain $T_{k}^{s}(t)=k(2 s-2) ! !\left(1-t^{2}\right)^{s / 2} C_{k-s}^{s}[t]$.

It is known [5] that for Gegenbauer polynomials $\int_{-1}^{1}\left(C_{k}^{s}[t]\right)^{2}\left(1-t^{2}\right)^{s-1 / 2} d t=$ $(2 s)_{k} \Gamma\left(\frac{1}{2}\right) \Gamma\left(s+\frac{1}{2}\right) /(k !(k+s) \Gamma(s))$. So, we can get

$$
\int_{-1}^{1}\left(T_{k}^{s}(t)\right)^{2}\left(1-t^{2}\right)^{-1 / 2} d t=k^{2}((2 s-2) ! !)^{2} \frac{(s+k-1) !}{(k-s) ! k((2 s-2) ! !)^{2}} \frac{\pi}{2} .
$$

Therefore,

$$
\int_{-1}^{1}\left(T_{k}^{s}(t)\right)^{2}\left(1-t^{2}\right)^{-1 / 2} d t=k \frac{(k+s-1) !}{(k-s) !} \frac{\pi}{2}
$$

whence using (16) we can obtain

$$
\int_{-1}^{1}\left(G_{k}^{s, n}(t)\right)^{2} \rho(t) d t=\frac{((2 s+n-3) ! !)^{2}}{(k+(n-2) / 2)(k+s+n-3) !(k-s) !} \frac{\pi}{2} .
$$

Combining (17) and (19) and using properties of the $\Gamma$-function, we get the necessary equality. The proof is completed.

Remark. Associated Chebyshev functions of the same order are orthogonal on the segment $[-1,1]$ with weight $\rho(t)=\left(1-t^{2}\right)^{-1 / 2}$, and the formula (18) holds.

Polynomials $G_{(\nu)}(x)$ for $n=2,3$ turn into well-known spherical functions [1].

Theorem 5. The scalar product of $G_{(\nu)}(x)$ on $G_{(\mu)}(x)$ in $L_{2}(\partial S)$ is equal to the product of scalar products $G_{\nu_{i}}^{\nu_{i+1}, n-i+1}(t)$ on $G_{\mu_{i}}^{\mu_{i+1}, n-i+1}(t)$ in $L_{2}(-1,1)$ with weights $\rho_{i}(t)=\left(1-t^{2}\right)^{(n-2-i) / 2}$ for $1 \leq i \leq n-1$.

Proof. The proof can be easily obtained by using definition (5) of $G_{(\nu)}(x)$, applying equality (6) and then using the property of $H_{k}^{s}\left(x_{(2)}\right)$ from (4). 
Theorem 6. The $G$-function $\bar{G}_{k}^{s, n}(t)$ normalized in $L_{2}(-1,1)$ with weight $\rho(t)=$ $\left(1-t^{2}\right)^{(n-3) / 2}$ satisfies the estimation

$$
\left|\bar{G}_{k}^{s, n}(t)\right| \leq 2^{k} \sqrt{(k+(n-2) / 2) 2^{n-3}}, \quad k \geq s .
$$

Proof. To evaluate $G_{k}^{s, n}(t)$ we need to rewrite it in a different form.

Using the known connection of $\Gamma$ - and $B$-functions, $B(p, s)=\Gamma(p) \Gamma(s) / \Gamma(p+s)$, and the equality $\Gamma(p+1)=p \Gamma(p)$ we can get

$$
\frac{1}{(n-1+2 s, 2)_{k}}=\frac{1}{(2 k-1) ! !} \frac{1}{B(1 / 2, s+(n-2) / 2)} \int_{0}^{1} \alpha^{k-1 / 2}(1-\alpha)^{s+(n-4) / 2} d \alpha .
$$

If $s \neq 0$ and $n \neq 2$, then from the definition of $G_{k}^{s, n}(t)(7)$ we obtain

$$
\begin{aligned}
G_{k}^{s, n}(t)=\frac{\left(1-t^{2}\right)^{s / 2}}{B(1 / 2, s+(n-2) / 2)} & \frac{1}{2} \int_{0}^{1}\left(\left(t+i \sqrt{\alpha\left(1-t^{2}\right)}\right)^{k-s, !}\right. \\
+ & \left.\left(t-i \sqrt{\alpha\left(1-t^{2}\right)}\right)^{k-s, !}\right)(1-\alpha)^{s+(n-4) / 2} \frac{d \alpha}{\sqrt{\alpha}},
\end{aligned}
$$

and therefore $\left|G_{k}^{s, n}(t)\right| \leq 1 /(k-s)$ !. From Theorem 4 we derive

$$
\left\|G_{k}^{s, n}(t)\right\|^{-2} \leq\left(k+\frac{n-2}{2}\right) \frac{(k+s+n-3) !(k-s) !}{((2 s+n-3) ! !)^{2}} .
$$

Thus, taking into account that $(2 s+n-3) ! \leq((2 s+n-3) ! !)^{2}$ we get

$$
\left.\left|\bar{G}_{k}^{s, n}(t)\right|^{2} \leq\left(k+\frac{n-2}{2}\right)\left(\begin{array}{c}
k+s+n-3 \\
k-3
\end{array}\right) \leq(k+(n-2) / 2)\right) 2^{2 k+n-3} .
$$

This implies that (20) is true. If $s=0$ and $n=2$, then $\left|G_{k}^{0,2}(t)\right|=\frac{1}{k !}\left|T_{k}(t)\right| \leq$ $1 / k$ ! and therefore, according to $(18),\left|\bar{G}_{k}^{0,2}(t)\right| \leq \sqrt{2 / \pi}<1$. Because $1<$ $\sqrt{k} 2^{k-1 / 2}$, the Theorem is true for this case too.

\section{ACKNOWLEDGMENT}

Research for this paper was supported in part by the Junior Faculty Development Program, which is funded by the Bureau of Educational and Cultural Affairs of the Department of State, under authority of the Fulbright-Hays Act of 1961 as amended, and administrated by the American Council for International Education: ACTR/ACCELS. The opinions expressed herein are the author's own and do not necessarily express the views of either ECA or the American Councils.

I would like also to thank Professor Paul E. Sacks for his valuable remarks.

\section{REFERENCES}

[1] E. M. Stein and G. Weiss, Introduction to Fourier analysis on Euclidean spaces, Princeton University Press, Princeton, NJ, 1971. MR 46:4102

[2] V. V. Karachik, Polynomial solutions to systems of partial differential equations with constant coefficients, Yokohama Mathematical Journal 47 (2) (2000), 121-142. MR 2001d:35031

[3] V. V. Karachik, On one set of orthogonal harmonic polynomials, Proc. Amer. Math. Soc. 126 (12) (1998), 3513-3519. MR 99g:33034

[4] A. Erdélyi, W. Magnus, F. Oberhettinger, and F. Tricomi, Higher Transcendental Functions, vol. 2, Based on notes left by Harry Bateman, McGraw-Hill, New York, 1953. MR 15:419i 
[5] E. D. Rainville, Special Functions, The Macmillan Company, New York, 1960. MR 21:6447

[6] V. S. Vladimirov, Uravneniya matematicheskoj fiziki, Fiziko-Matematicheskaya Literatura, Moskva, 2000 (Russian).

148, Behterev St., Apt. 6, 700105, Tashkent, Uzbekistan

E-mail address: karachik@uwed.freenet.uz

Current address: Institute of Cybernetics, Uzbek Academy of Sciences, 34, F. Hodzhaev St., 700125, Tashkent, Uzbekistan

E-mail address: karachik@tkt.uz 